\title{
Test for parameter changes in generalized random coefficient autoregressive model
}

\author{
Zhi-Wen Zhao ${ }^{1 *}$, De-Hui Wang ${ }^{2}$ and Cui-Xin Peng ${ }^{3}$
}

${ }^{*}$ Correspondence:

zhaozhiwen@126.com

${ }^{1}$ College of Mathematics, Jilin

Normal University, Siping, 136000,

P.R. China

Full list of author information is

available at the end of the article

\begin{abstract}
In this paper, we study the problem of testing for parameter changes in generalized random coefficient autoregressive model (GRCA). The testing method is based on the monitoring scheme proposed by Na et al. (Stat. Methods Appl. 20:171-199, 2011), and the test statistic relies on the conditional least-squares estimator of an unknown parameter. Furthermore, under mild conditions, we obtain the asymptotic property of the test statistic. Some simulation studies are also conducted to investigate the finite sample performances of the proposed test.
\end{abstract}

MSC: Primary 62M10; secondary 91B62

Keywords: monitoring parameter changes; conditional least-squares estimation; asymptotic variance; generalized random coefficient autoregressive model

\section{Introduction}

Consider the following one-order generalized random coefficient autoregressive model $(\operatorname{GRCA}(1))$ :

$$
Y_{t}=\Phi_{t} Y_{t-1}+\varepsilon_{t}, \quad t=0, \pm 1, \pm 2, \ldots
$$

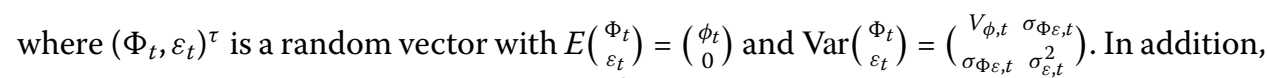
$\left(\Phi_{t}, \varepsilon_{t}\right)^{\tau}$ is assumed to be independent of $\mathscr{F}_{t-1}=\sigma\left(Y_{t-1}, Y_{t-2}, \ldots\right)$.

The model (1.1) was first introduced by Hwang and Basawa [1]. When $\Phi_{t}$ and $\varepsilon_{t}$ are mutually independent, the model (1.1) becomes the random coefficient autoregressive model (RCAR), and when $V_{\phi}=0$, the model (1.1) becomes the usual autoregressive model. Furthermore, the model (1.1) also includes the Markovian bilinear model (see, e.g., [2, 3]), the generalized Markovian bilinear model, and the random coefficient exponential autoregressive model (see [4] for more information) as special cases.

GRCA is designed for investigating the result of random perturbations of a dynamical system in engineering and economic data, and it has become one of the important models in the nonlinear time series context. In several recent years, GRCA has been studied by many authors. For instance, Hwang and Basawa [5] established the local asymptotic normality of a class of generalized random coefficient autoregressive processes. Lee [6] studied the problem of testing the constancy of the coefficient. Moreover, Carrasco and Chen [7] provided tractable sufficient conditions that simultaneously imply strict stationarity, finiteness of higher-order moments, and $\beta$-mixing with geometric decay rates. In this paper, we consider the problem of testing for parameter changes in GRCA.

@2014 Zhao et al:; licensee Springer. This is an Open Access article distributed under the terms of the Creative Commons Attribution License (http://creativecommons.org/licenses/by/2.0), which permits unrestricted use, distribution, and reproduction in any medium, provided the original work is properly cited. 
The change-point problem has a long history and began with i.i.d. samples (see, e.g., [813]). Observing that time series often suffer from structural changes, statisticians started the study of the change-point problem for economic time series models (see, e.g., [14, 15]). Recently, the change-point problem has become very popular in economic time series. Lee and Park [16] considered the monitoring process in time series regression models with nonstationary regressors. Gombay and Serban [17] proposed sequential tests to detect an abrupt change in any parameter, or in any collection of parameters of an autoregressive time series model. By using the cumulative sum test, Kang and Lee [18] studied the problem of testing for a parameter change in a first-order random coefficient integer-valued autoregressive model. Moreover, $\mathrm{Na}$ et al. [19] also designed the monitoring procedure in general time series models and applied it to the changes of the autocovariances of linear processes, GARCH parameters, and underlying distributions.

In order to monitor the parameter changes in generalized random coefficient autoregressive model, we employ the monitoring scheme proposed by $\mathrm{Na}$ et al. [19]. The test statistic relies on the conditional least-squares estimator of an unknown parameter, and under mild conditions we also obtain the asymptotic property of the test statistic.

The rest of this paper is organized as follows: In Section 2, we introduce the methodology and the main results. Simulation results are reported in Section 3. A real data analysis is given in Section 4. Section 5 provides the proofs of the main results.

Throughout this paper, we denote $p$-dimensional standard Brown motion by $\left\{W_{p}(s), s \geq\right.$ $0\}$. The symbols ' $\stackrel{d}{\rightarrow}$ ' and ' $\stackrel{p}{\rightarrow}$ ' denote convergence in distribution and convergence in probability, respectively, and convergence 'almost surely' is written as 'a.s'.

\section{Methodology and main results}

$\mathrm{Na}$ et al. [19] proposed a monitoring scheme of detecting parameter changes for general time series models. In what follows, we will first introduce this monitoring procedure briefly, and then we give our main results.

Let $\left\{Y_{t}\right\}$ be a time series and $\Theta$ be unknown parameter which will be examined for the parameter constancy. We assume that $\Theta$ is a constant for the historical data $Y_{1}, Y_{2}, \ldots, Y_{T}$. Here, we wish to test the following hypotheses based on the estimator $\hat{\Theta}_{T}$ of $\Theta$ :

$\mathrm{H}_{0}: \Theta$ does not change over time $t>T$ versus $\mathrm{H}_{1}: \Theta$ changes at some time $t>T$.

We assume that, under $\mathrm{H}_{0}, \hat{\Theta}_{T}$ is a $\sqrt{T}$-consistent estimator of $\Theta$ based on $Y_{1}, Y_{2}, \ldots, Y_{T}$, its asymptotic variance-covariance matrix $\Sigma$ is nonsingular and $\hat{\Sigma}_{T}$ is the consistent estimator of $\Sigma$ based on $Y_{1}, Y_{2}, \ldots, Y_{T}$. We can then define

$$
\tau(T)=\inf \left\{k>T:\left\|\hat{\Sigma}_{T}^{-\frac{1}{2}}\left(\hat{\Theta}_{k}-\hat{\Theta}_{T}\right)\right\| \geq \frac{1}{\sqrt{T}} g\left(\frac{k}{T}\right)\right\}
$$

where $\hat{\Theta}_{k}$ is the estimator of $\Theta$ at time lag $k>T$ based on $Y_{1}, Y_{2}, \ldots, Y_{k},\|\cdot\|$ denotes a norm on $R^{p}$, and $g(s)(s \in(1, \infty))$ is a given boundary function.

If $\tau(T)$ is finite, we reject $\mathrm{H}_{0}$. In actual practice, if there exists $k \in(T, T+q)$, with $q=$ $2 T, 3 T, 5 T, 10 T$ etc., such that $\left\|\hat{\Sigma}_{T}^{-\frac{1}{2}}\left(\hat{\Theta}_{k}-\hat{\Theta}_{T}\right)\right\| \geq \frac{1}{\sqrt{T}} g\left(\frac{k}{T}\right)$, then we reject $\mathrm{H}_{0}$. Meanwhile, the boundary function $g$ is chosen to satisfy

$$
\lim _{T \rightarrow \infty} P_{\mathrm{H}_{0}}\{\tau(T)<\infty\}=\alpha
$$


for a given $\alpha \in(0,1)$ and

$$
\lim _{T \rightarrow \infty} P_{\mathrm{H}_{1}}\{\tau(T)<\infty\}=1
$$

Further, under $\mathrm{H}_{0}$, suppose that the estimator $\hat{\Theta}_{k}(k=T, T+1, \ldots)$ satisfies the following conditions:

$\left(A_{1}\right) \hat{\Theta}_{k}$ can be decomposed as

$$
\hat{\Theta}_{k}=\Theta_{0}+\frac{1}{k} \sum_{t=1}^{k} l_{t}+\triangle_{k}
$$

where $\Theta_{0}$ is a true value of $\Theta$ under $\mathrm{H}_{0},\left\{l_{t}=\left(l_{1 t}, \ldots, l_{p t}\right)^{\tau}, t \geq 1\right\}$ is a sequence of $p$-dimensional random vectors and $\triangle_{k}=\left(\triangle_{1 k}, \ldots, \triangle_{p k}\right)^{\tau}$ are negligible terms.

$\left(\mathrm{A}_{2}\right)$ There exists a $p$-dimensional standard Brownian motion $\left\{W_{p}(s), s \geq 0\right\}$ such that, for some $0<\lambda<\frac{1}{2}$

$$
\sum_{t=1}^{k} l_{t}-\Sigma^{-\frac{1}{2}} W_{p}(k)=O\left(k^{\lambda}\right) \quad \text { a.s. }
$$

$\left(\mathrm{A}_{3}\right)$ For each $1 \leq i \leq p, \sqrt{T} \sup _{k \geq T}\left|\triangle_{i, k}\right|=o_{p}(1)$

Remark 1 Condition $\left(\mathrm{A}_{2}\right)$ holds for zero mean stationary martingale difference sequence $\left\{l_{t}, t \geq 1\right\}$ and at this point $\Sigma=E\left(l_{1} l_{1}^{\tau}\right)($ see $[20,21])$.

Based on the above conditions, we have the following lemmas.

Lemma 2.1 Suppose that $\left(\mathrm{A}_{1}\right)-\left(\mathrm{A}_{3}\right)$ hold. If $g(s)=c g_{1}(s), s \in(1, \infty), c$ is a positive constant and $g_{1}$ is a given continuous real-valued function with $\inf _{s \in(1, \infty)} g_{1}(s)>0$, then

$$
\begin{aligned}
\lim _{T \rightarrow \infty} P_{\mathrm{H}_{0}}\{\tau(T)<\infty\} & =P\left\{\left\|W_{p}(s)-s W_{p}(1)\right\| \geq s g(s) \text { for some } s>1\right\} \\
& =P\left\{\left\|W_{p}(s)\right\| \geq g(1 /(1-s)) \text { for some } 0<s<1\right\} \\
& =P\left\{\sup _{s \in(0,1)} \frac{\left\|W_{p}(s)\right\|}{g_{1}(1 /(1-s))} \geq c\right\} .
\end{aligned}
$$

Particularly, if $g(s)=c$, then

$$
\lim _{T \rightarrow \infty} P_{\mathrm{H}_{0}}\{\tau(T)<\infty\}=P\left\{\sup _{s \in(0,1)}\left\|W_{p}(s)\right\| \geq c\right\}
$$

Lemma 2.1 is due to Na et al. [19]. Moreover, $\left(\mathrm{A}_{1}\right)-\left(\mathrm{A}_{3}\right)$ and the consistency of $\hat{\Sigma}_{T}$ indicate that

$$
\frac{[T s]}{\sqrt{T}} \hat{\Sigma}_{T}^{-\frac{1}{2}}\left(\hat{\theta}_{[T s]}-\hat{\theta}_{T}\right) \stackrel{w}{\rightarrow} W_{p}(s)-s W_{p}(1), \quad s \in[1, \infty)
$$

as $T \rightarrow \infty$. Thus, by using Theorem 3.4 of Chu et al. [22], we have the following lemma. 
Lemma 2.2 Suppose that $\left(\mathrm{A}_{1}\right)-\left(\mathrm{A}_{3}\right)$ hold. If $g(s)=\sqrt{\frac{s-1}{s}\left(e^{2}+\ln \left(\frac{s}{s-1}\right)\right)}, s \in(1, \infty)$ and $\|\cdot\|_{\infty}$ is the maximum norm, then

$$
\begin{aligned}
\lim _{T \rightarrow \infty} P_{\mathrm{H}_{0}}\{\tau(T)<\infty\} & =P\left\{\left\|W_{p}(s)-s W_{p}(1)\right\|_{\infty} \geq s g(s) \text { for some } s>1\right\} \\
& =1-[1-2\{1-\Phi(e)+e \phi(e)\}]^{p},
\end{aligned}
$$

where $e$ is a constant, $\phi$ and $\Phi$ denote the standard normal density and distribution functions, respectively.

Below, we use the above method to detect parameter changes in the model (1.1). Suppose that $\sigma_{\varepsilon, t}^{2}=\sigma_{\varepsilon, 0}^{2}, \sigma_{\Phi \varepsilon, t}=\sigma_{\Phi \varepsilon, 0}, V_{\phi, t}=V_{\phi, 0}, t=1,2, \ldots$ Moreover, we assume that $\phi_{t}=\phi_{0}$ for $t=1, \ldots, T$. Consider the following hypothesis test:

$\mathrm{H}_{0}: \phi_{t}=\phi_{0}, t>T$ versus $\mathrm{H}_{1}: \phi_{t}$ changes at some $t>T$.

Before we state our main results, we list some regular conditions used in this paper.

$\left(C_{1}\right)$ The distributions of $\Phi_{t}$ and $\varepsilon_{t}$ are absolutely continuous with respect to the Lebesgue measure on $R^{1}$ and their densities are strictly positive on some neighborhood of 0 .

(C $\left.\mathrm{C}_{2}\right) \theta_{0}=\phi_{0}^{2}+V_{\phi, 0}<1$.

$\left(\mathrm{C}_{3}\right) E\left(\Phi_{t}^{6}\right)<1$ and $E\left(\varepsilon_{t}^{6}\right)<\infty$.

Remark 2 It is shown by Theorem 2.1 of Hwang and Basawa [1] that, under $\left(C_{2}\right),\left\{Y_{t}, t \geq 1\right\}$ is stationary and ergodic.

In order to establish the test statistic we need to obtain the consistent estimator of parameter $\phi_{0}$ under $\mathrm{H}_{0}$. Below we further assume that conditions $\left(\mathrm{C}_{1}\right)-\left(\mathrm{C}_{3}\right)$ hold. Based on the recorded data $\left\{Y_{0}, \ldots, Y_{k}\right\}$, the conditional least-squares estimator $\hat{\phi}_{k}$ of $\phi_{0}$ is obtained by minimizing

$$
S=\sum_{t=1}^{k}\left(Y_{t}-E\left(Y_{t} \mid Y_{t-1}\right)\right)^{2}
$$

with respect to $\phi$. Substituting $E\left(Y_{t} \mid Y_{t-1}\right)=\phi Y_{t-1}$ in $S$ and solving $d S / d \phi=0$ for $\phi$, we obtain

$$
\hat{\phi}_{k}=\left(\sum_{t=1}^{k} Y_{t-1} Y_{t-1}\right)^{-1}\left(\sum_{t=1}^{k} Y_{t} Y_{t-1}\right) .
$$

Under the conditions $\left(C_{2}\right)$ and $\left(C_{3}\right)$, the estimator $\hat{\phi}_{k}$ is consistent and asymptotically normal, and its asymptotic variance $J=\sigma_{\varepsilon, 0}^{-2}\left(1-\theta_{0}\right)^{2}\left(\sigma_{\varepsilon, 0}^{2} E Y_{0}^{2}+2 \sigma_{\Phi \varepsilon, 0} E Y_{0}^{3}+\left(\theta_{0}-\phi_{0}^{2}\right) E Y_{0}^{4}\right)$ (see Hwang and Basawa [1]).

In what follows, we consider an estimate of $J$. By the ergodic theorem, it is easy to find that, for any integer $l>1, \frac{1}{n} \sum_{t=1}^{n} Y_{t-1}^{l}$ is the consistent estimator of $E Y_{0}^{l}$. Therefore, in order to obtain the consistent estimator $\hat{J}_{k}$ of $J$, we only need to obtain the consistent estimator of $\Upsilon=\left(\theta_{0}, \sigma_{\varepsilon, 0}^{2}, \sigma_{\Phi \varepsilon, 0}\right)^{\tau}$. 
We now consider an estimate of $\Upsilon$. A conditional least-squares estimator $\hat{\Upsilon}_{k}$ of $\Upsilon$ can be obtained by minimizing

$$
Q(\Upsilon)=\sum_{t=1}^{k}\left(Y_{t}^{2}-E\left(Y_{t}^{2} \mid Y_{t-1}\right)\right)^{2}=\sum_{t=1}^{k}\left(Y_{t}^{2}-N_{t}^{\tau} \Upsilon\right)^{2}
$$

where $N_{t}=\left(Y_{t-1}^{2}, 1,2 Y_{t-1}\right)^{\tau}$. Solving $d Q / d \Upsilon=0$ for $\Upsilon$, we obtain

$$
\hat{\Upsilon}_{k}=\left(\sum_{t=1}^{k} N_{t} N_{t}^{\tau}\right)^{-1} \sum_{t=1}^{k} Y_{t}^{2} N_{t}
$$

The following lemma indicates that $\hat{\Upsilon}_{k}$ is the consistent estimator of $\Upsilon$.

Lemma 2.3 Suppose that $\left(\mathrm{C}_{2}\right)$ and $\left(\mathrm{C}_{3}\right)$ hold. Then, under $\mathrm{H}_{0}$, we have

$$
\hat{\Upsilon}_{k} \stackrel{\text { a.s. }}{\rightarrow} \Upsilon \text {. }
$$

After obtaining the consistent estimator of $\phi_{0}$ and $J$, we can establish the following test statistics:

$$
\tau_{1}(T)=\inf \left\{k>T:\left\|\hat{J}_{T}^{-\frac{1}{2}}\left(\hat{\phi}_{k}-\hat{\phi}_{T}\right)\right\| \geq \frac{1}{\sqrt{T}} g\left(\frac{k}{T}\right)\right\} .
$$

For the test statistics $\tau_{1}(T)$, we have the following results.

Theorem 2.1 Suppose that $\left(\mathrm{C}_{1}\right)-\left(\mathrm{C}_{3}\right)$ hold.

(i) If $g(s)=c g_{1}(s), s \in(1, \infty), c$ is a positive constant, and $g_{1}$ is a given continuous real-valued function with $\inf _{s \in(1, \infty)} g_{1}(s)>0$, then

$$
\lim _{T \rightarrow \infty} P_{\mathrm{H}_{0}}\left\{\tau_{1}(T)<\infty\right\}=P\left\{\sup _{s \in(0,1)} \frac{\left\|W_{1}(s)\right\|}{g_{1}(1 /(1-s))} \geq c\right\} .
$$

Particularly, if $g(s)=c$ and $\|\cdot\|=\|\cdot\|_{2}$, then

$$
\lim _{T \rightarrow \infty} P_{\mathrm{H}_{0}}\left\{\tau_{1}(T)<\infty\right\}=P\left\{\sup _{s \in(0,1)}\left|W_{1}(s)\right| \geq c\right\} .
$$

(ii) If $g(s)=\sqrt{\frac{s-1}{s}\left(e^{2}+\ln \left(\frac{s}{s-1}\right)\right)}, s \in(1, \infty)$, and $\|\cdot\|=\|\cdot\|_{\infty}$, then

$$
\lim _{T \rightarrow \infty} P_{\mathrm{H}_{0}}\left\{\tau_{1}(T)<\infty\right\}=2-2 \Phi(e)+2 e \phi(e) .
$$

Remark 3 If $g(s)=c$ and $\|\cdot\|=\|\cdot\|_{2}$, we have

$$
\begin{aligned}
\tau_{1}(T) & =\inf \left\{k>T:\left\|\hat{J}_{T}^{-\frac{1}{2}}\left(\hat{\phi}_{k}-\hat{\phi}_{T}\right)\right\|_{2} \geq \frac{c}{\sqrt{T}}\right\} \\
& =\inf \left\{k>T:\left|\hat{J}_{T}^{-\frac{1}{2}}\left(\hat{\phi}_{k}-\hat{\phi}_{T}\right)\right| \geq \frac{c}{\sqrt{T}}\right\} \\
& =\inf \left\{k>T: T \hat{J}_{T}^{-1}\left(\hat{\phi}_{k}-\hat{\phi}_{T}\right)^{2} \geq c^{2}\right\} .
\end{aligned}
$$


By (i) of Theorem 2.1, we can determine the constant $c$ for any significance level $\alpha \in(0,1)$. In fact, since $\lim _{T \rightarrow \infty} P_{\mathrm{H}_{0}}\left\{\tau_{1}(T)<\infty\right\}=P\left\{\sup _{s \in(0,1)}\left|W_{1}(s)\right| \geq c(\alpha)\right\}=\alpha$, where $c(\alpha)$ is the $1-\alpha$ quantile point of $\sup _{s \in(0,1)}\left|W_{1}(s)\right|$, we thus have $c=c(\alpha)$. When $p=1$, we have $c=7.78$ for $\alpha=0.1$. Moreover, if $g(s)=\sqrt{\frac{s-1}{s}\left(e^{2}+\ln \left(\frac{s}{s-1}\right)\right)}, s \in(1, \infty)$, and $\|\cdot\|=\|\cdot\|_{\infty}$, then when $p=1$, we have $e^{2}=1.488$ for the nominal level $\alpha=0.1$.

\section{Simulation results}

In this section, we evaluate the performance of the monitoring test through a simulation study.

Consider the following model:

$$
Y_{t}=\left(\phi+\alpha \varepsilon_{t}\right) Y_{t-1}+\varepsilon_{t}
$$

where $\left\{\varepsilon_{t}\right\}$ is i.i.d. normally distributed with mean 0 and variance 1 .

We compare the performance of testing methods (i) and (ii) in Theorem 2.1. For the testing method (i), we take $g(s)=c$ and $\|\cdot\|=\|\cdot\|_{2}$. In the actual simulation, we reject $\mathrm{H}_{0}$ if there exists $k \in(T, T+q)$ such that $\left\|\hat{J}_{T}^{-\frac{1}{2}}\left(\hat{\phi}_{k}-\hat{\phi}_{T}\right)\right\| \geq \frac{1}{\sqrt{T}} g\left(\frac{k}{T}\right)$, where the horizon $q=2 T, 3 T, 5 T$ and $10 T$, and $T=100,200,300,500$ and 1,000. In each simulation, 1,000 observations are discarded to remove initialization effects and a repetition number of 1,000 is utilized.

In the first simulation, we calculate the probability of rejecting the null hypothesis when it is true at the nominal level $\alpha=0.1$. The results of the simulations are presented in Table 1 , and the figures in parentheses are those for a constant function test.

From Table 1, we see that the test with the boundary function in (ii) of Theorem 2.1 has superiority over that with the constant boundary function. We can also see that the empirical sizes of these two tests tend to decrease as the historical sample size $T$ increases and increase as $q$ increases. But even if $T$ is small or $q$ is large, the empirical sizes of these two testing methods are still very close to the nominal level.

The second simulation study is designed to examine the power. We calculate the probability of rejecting the null hypothesis when the alternative hypothesis is true at the nominal level $\alpha=0.1$. To do this, we consider the alternative hypotheses as follows:

$\mathrm{H}_{1}$ : A change occurs from $\phi_{t}=0.1, \alpha=0.3$ to $\phi_{t}=0.7, \alpha=0.3$;

$\tilde{\mathrm{H}}_{1}$ : A change occurs from $\phi_{t}=0.7, \alpha=0.3$ to $\phi_{t}=0.1, \alpha=0.3$.

In all cases, the changes are assumed to occur at $k^{*}=0.3 T$ and $k^{*}=0.8 T$. The results of the simulations are presented in Table 2, and the figures in parentheses are those for the constant function test.

From Table 2, we see that the probability of rejecting the null hypothesis when the alternative hypothesis is true tends to increase as $T$ increases or $q$ increases, and the test with the constant boundary function tends to detect changes more efficiently than the test in (ii) of Theorem 2.1. It can also be seen from Table 2 that if $\phi_{t}$ changes from a smaller number to a larger number, then these two test approaches perform very well. Although these two test approaches tend to mistakenly detect changes when $\phi_{t}$ changes from a larger number to a smaller number when $T$ and $q$ are small, both these two tests still perform well when $T$ and $q$ are large. Therefore, when we use the above methods to detect the parameter changes, we suggest that one estimate the parameter firstly. If the parameter is small, we can use a smaller $q$ or $T$. 
Table 1 Empirical sizes

\begin{tabular}{|c|c|c|c|c|c|}
\hline$\phi_{t}$ & $T$ & $=2 T$ & $q=3 T$ & $q=5 T$ & $q=10 T$ \\
\hline 0.1 & $\begin{array}{r}100 \\
200 \\
300 \\
500 \\
1,000\end{array}$ & $\begin{array}{l}0.084(0.179) \\
0.050(0.167) \\
0.061(0.162) \\
0.071(0.139) \\
0.056(0.153)\end{array}$ & $\begin{array}{l}0.084(0.207) \\
0.061(0.181) \\
0.057(0.207) \\
0.050(0.184) \\
0.058(0.185)\end{array}$ & $\begin{array}{l}0.070(0.255) \\
0.046(0.228) \\
0.056(0.229) \\
0.054(0.242) \\
0.055(0.224)\end{array}$ & $\begin{array}{l}0.096(0.28 \\
0.049(0.24 \\
0.053(0.25 \\
0.059(0.25 \\
0.065(0.30\end{array}$ \\
\hline 0.3 & $\begin{array}{r}100 \\
200 \\
300 \\
500 \\
1,000\end{array}$ & & & & $\begin{array}{l}0.103(0.26 \\
0.078(0.25 \\
0.069(0.26 \\
0.073(0.27) \\
0.069(0.26\end{array}$ \\
\hline 0. & $\begin{array}{r}100 \\
200 \\
300 \\
500 \\
1,000\end{array}$ & $\begin{array}{l}0.090(0.160) \\
0.086(0.121) \\
0.087(0.149) \\
0.071(0.137) \\
0.073(0.118)\end{array}$ & & & $\begin{array}{l}0.101(0.23 \\
0.078(0.19 \\
0.060(0.20 \\
0.073(0.20 \\
0.069(0.22\end{array}$ \\
\hline 0. & $\begin{array}{r}100 \\
200 \\
300 \\
500 \\
1,000\end{array}$ & $\begin{array}{l}0.078(0.138) \\
0.063(0.098) \\
0.076(0.096) \\
0.052(0.078) \\
0.041(0.081)\end{array}$ & $\begin{array}{l}0.055(0.092) \\
0.073(0.090) \\
0.064(0.083) \\
0.055(0.089) \\
0.069(0.081)\end{array}$ & $\begin{array}{l}0.111(0.184) \\
0.065(0.097) \\
0.057(0.110) \\
0.067(0.108) \\
0.049(0.117)\end{array}$ & $\begin{array}{l}0.094(0.19) \\
0.058(0.11 \\
0.054(0.09 \\
0.056(0.11) \\
0.063(0.11\end{array}$ \\
\hline 0.5 & $\begin{array}{r}100 \\
200 \\
300 \\
500 \\
1,000\end{array}$ & $\begin{array}{l}0.067(0.100) \\
0.036(0.042) \\
0.037(0.060) \\
0.046(0.056) \\
0.033(0.042)\end{array}$ & $\begin{array}{l}0.049(0.063) \\
0.041(0.052) \\
0.044(0.057) \\
0.038(0.068) \\
0.047(0.063)\end{array}$ & $\begin{array}{l}0.078(0.117) \\
0.049(0.071) \\
0.051(0.060) \\
0.032(0.064) \\
0.039(0.065)\end{array}$ & $\begin{array}{l}0.098(0.16 \\
0.041(0.08 \\
0.044(0.06 \\
0.049(0.07 \\
0.035(0.08\end{array}$ \\
\hline
\end{tabular}

Table 2 Empirical powers

\begin{tabular}{cccccc}
\hline $\boldsymbol{T}$ & $\boldsymbol{k}^{*}$ & $\boldsymbol{q}=\mathbf{2 T}$ & $\boldsymbol{q}=\mathbf{3 T}$ & $\boldsymbol{q}=\mathbf{5 T}$ & $\boldsymbol{q}=\mathbf{1 0 T}$ \\
\hline$\phi_{t}=0.1, \alpha=0.3 \rightarrow \phi_{t}=0.7, \alpha=0.3$ & & & \\
100 & $0.3 T$ & $0.781(0.962)$ & $0.876(0.992)$ & $0.908(0.995)$ & $0.952(1.000)$ \\
& $0.8 T$ & $0.575(0.890)$ & $0.756(0.970)$ & $0.857(0.993)$ & $0.923(0.998)$ \\
200 & $0.3 T$ & $0.965(0.998)$ & $0.991(1.000)$ & $0.996(1.000)$ & $0.999(1.000)$ \\
& $0.8 T$ & $0.846(0.993)$ & $0.961(1.000)$ & $0.998(1.000)$ & $1.000(1.000)$ \\
300 & $0.3 T$ & $0.960(0.998)$ & $0.999(1.000)$ & $0.999(1.000)$ & $1.000(1.000)$ \\
& $0.8 T$ & $0.958(1.000)$ & $0.995(1.000)$ & $1.000(1.000)$ & $1.000(1.000)$ \\
500 & $0.3 T$ & $1.000(1.000)$ & $1.000(1.000)$ & $1.000(1.000)$ & $1.000(1.000)$ \\
& $0.8 T$ & $0.999(1.000)$ & $1.000(1.000)$ & $1.000(1.000)$ & $1.000(1.000)$ \\
1,000 & $0.3 T$ & $1.000(1.000)$ & $1.000(1.000)$ & $1.000(1.000)$ & $1.000(1.000)$ \\
& $0.8 T$ & $1.000(1.000)$ & $1.000(1.000)$ & $1.000(1.000)$ & $1.000(1.000)$ \\
$\phi_{t}=0.7, \alpha=0.3 \rightarrow \phi t=0.1, \alpha=0.3$ & & \\
100 & $0.3 T$ & $0.164(0.487)$ & $0.206(0.671)$ & $0.288(0.828)$ & $0.385(0.898)$ \\
& $0.8 T$ & $0.091(0.266)$ & $0.130(0.461)$ & $0.178(0.702)$ & $0.975(1.000)$ \\
200 & $0.3 T$ & $0.359(0.971)$ & $0.193(0.846)$ & $0.517(0.974)$ & $0.974(0.999)$ \\
& $0.8 T$ & $0.353(0.976)$ & $0.229(0.856)$ & $0.518(0.979)$ & $0.972(1.000)$ \\
300 & $0.3 T$ & $0.382(0.972)$ & $0.227(0.839)$ & $0.518(0.979)$ & $0.977(1.000)$ \\
500 & $0.8 T$ & $0.372(0.968)$ & $0.219(0.859)$ & $0.510(0.866)$ & $0.963(0.999)$ \\
500 & $0.3 T$ & $0.371(0.967)$ & $0.806(0.990)$ & $0.936(0.996)$ & $0.971(0.999)$ \\
1,000 & $0.8 T$ & $0.376(0.974)$ & $0.912(1.000)$ & $0.994(1.000)$ & $1.000(1.000)$ \\
& $0.3 T$ & $0.385(0.970)$ & $0.909(0.999)$ & $0.997(1.000)$ & $1.000(1.000)$ \\
& $0.8 T$ & $0.394(0.970)$ & $0.892(1.000)$ & $0.995(1.000)$ & $1.000(1.000)$ \\
\hline
\end{tabular}

\section{Real data analysis}

In this section, we illustrate how our method can be applied to a practical example. Specifically, we apply the above test methods to model offence data, which are the best indicator of crimes with victims such as robbery, sex offence, homicide, drugs, burglary, arson, etc. 
We mainly pay attention to drugs data series. The data are available on-line at the forecasting principles site (http://www.forecastingprinciples.com/index.php?option=com content\&view=article\&id=47\&Itemid=250). An observation of the time series represents a count of drugs reported in the police car beat in Pittsburgh, during one month. The data consist of 120 observations, starting in January 1992 and ending in December 2001. The data are denoted $y_{1}, y_{2}, \ldots, y_{120}$. Figure 1 is the sample path plot for the real data $y_{t}$, $t=1,2, \ldots, 120$.

The sample path plot reveals nonstationarity. Therefore, let $x_{t}=y_{t}-y_{t-1}$. The sample path plot, the autocorrelation function (ACF), and the partial autocorrelation function (PACF) for the differenced series $x_{t}$ is given in Figures 2, 3, and 4, respectively. From Figure 2, we can see that $x_{t}$ is from a stationary series. From Figures 3 and 4 , we can see that $x_{t}$ may come from an GRCA(1) process. Therefore, we consider a model of the data series $x_{t}$ by using the following:

$$
x_{t}=\left(\phi+\alpha \varepsilon_{t}\right) x_{t-1}+\varepsilon_{t},
$$

where $\left\{\varepsilon_{t}\right\}$ is for i.i.d. random variables.

We assume that $\phi$ is a constant for the historical data $x_{1}, x_{2}, \ldots, x_{50}$. Then we test the following hypotheses:

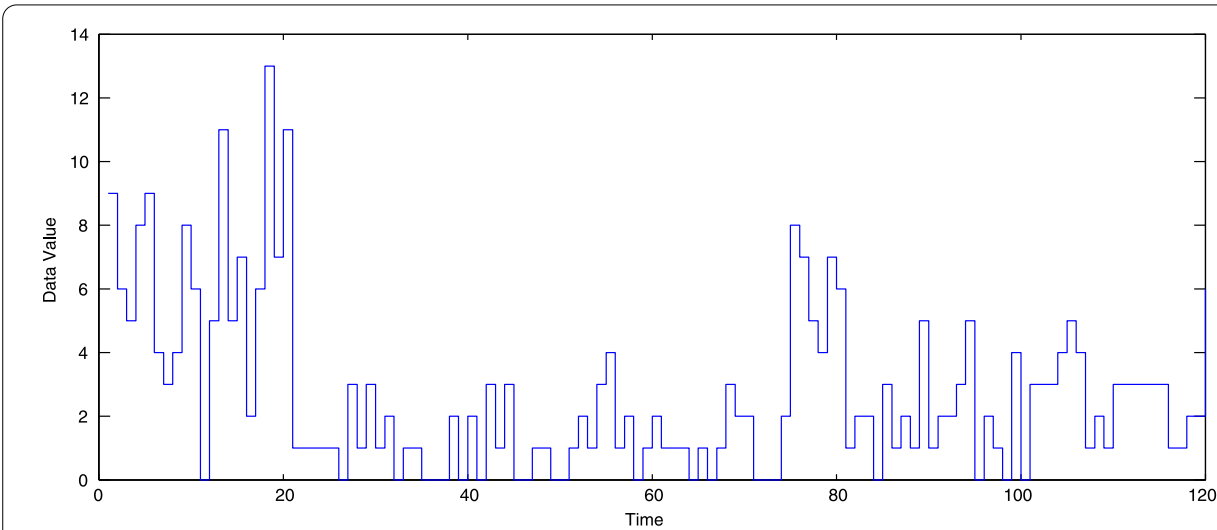

Figure 1 The sample path of real data $y_{t}$.

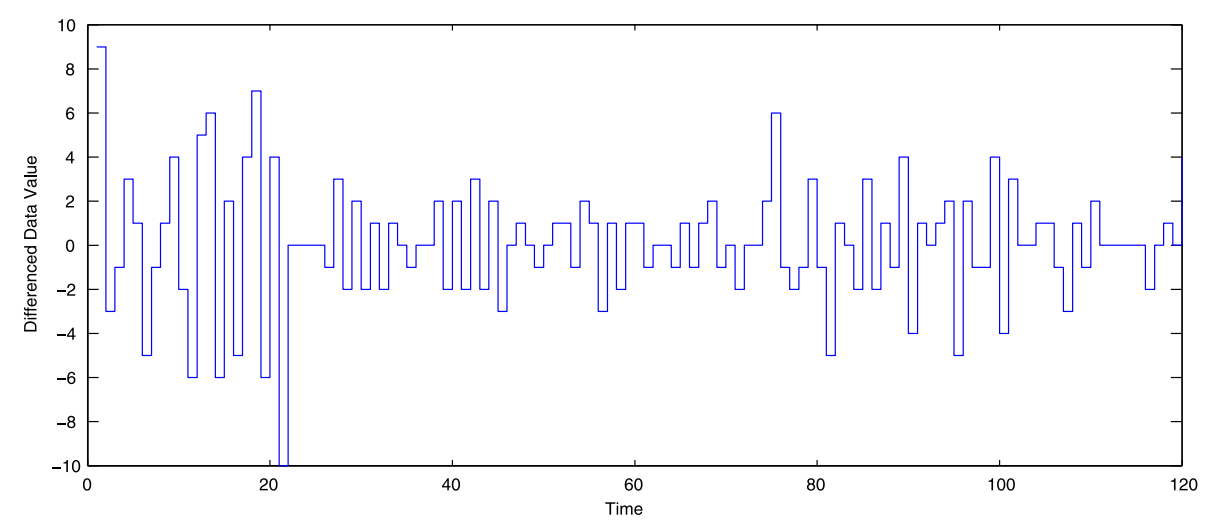

Figure 2 The sample path of differenced series $x_{t}$ for the real data. 


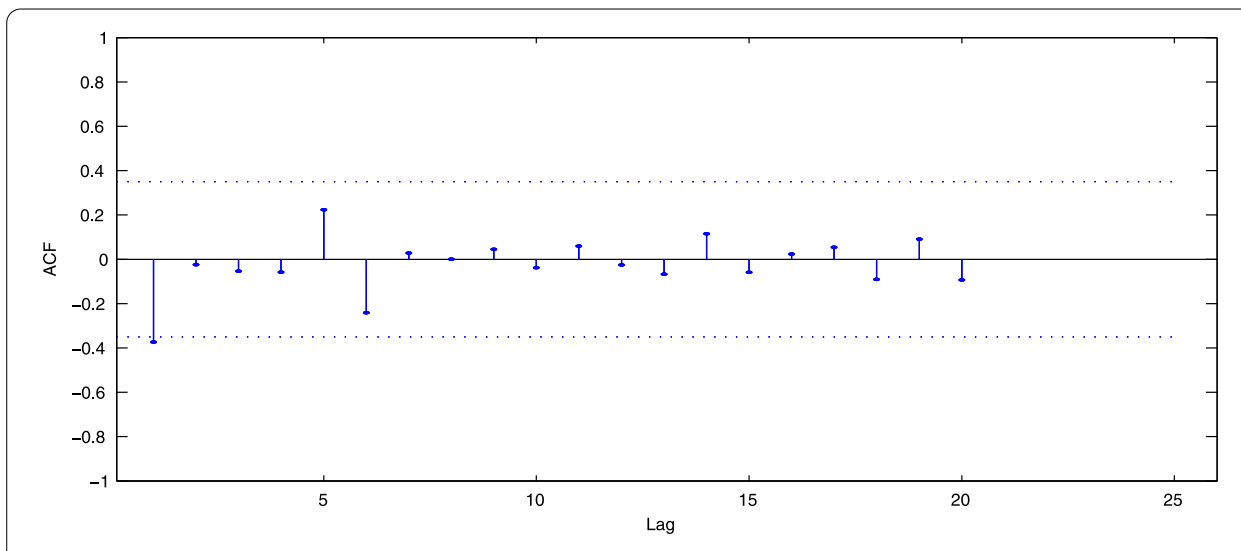

Figure 3 ACF plot of differenced series $x_{t}$.

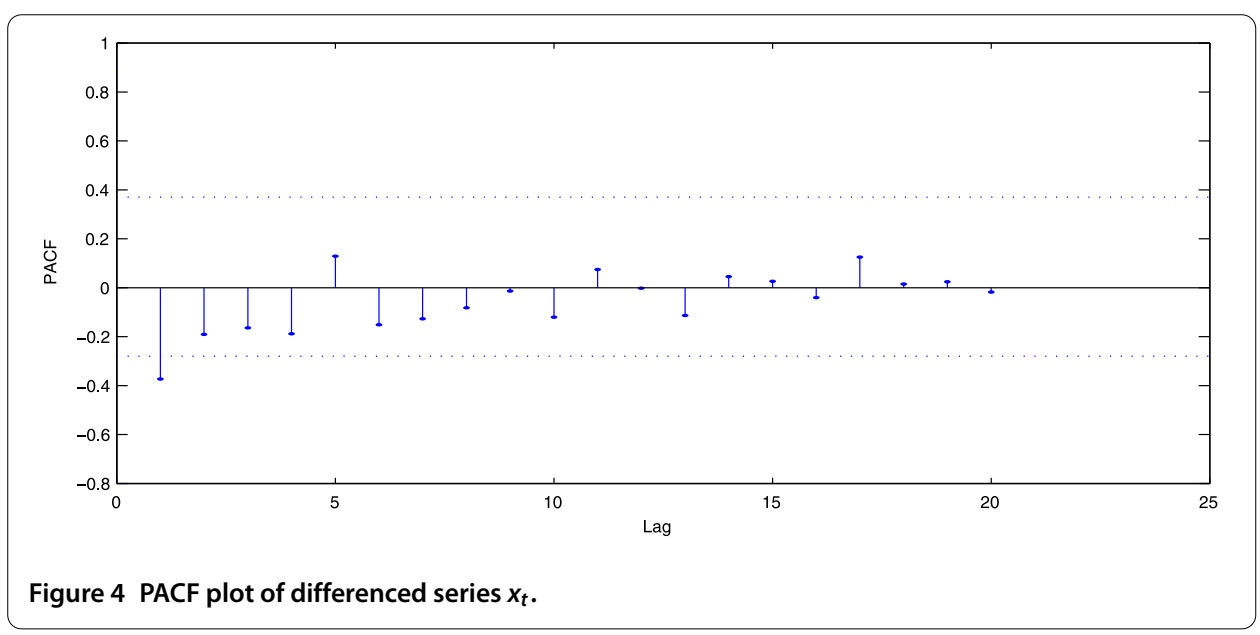

$\mathrm{H}_{0}: \phi$ does not change over time $t>50$ versus $\mathrm{H}_{1}: \phi$ changes at some time $t>50$.

Based on the historical data $x_{1}, x_{2}, \ldots, x_{50}$, testing methods (i) and (ii) in Theorem 2.1 both accept the null hypothesis. That is to say, $\phi$ does not change over time $t>50$. Further, we use the data $x_{1}, x_{2}, \ldots, x_{120}$ to estimate the unknown parameters $\phi$ and $\alpha$, respectively. Therefore, we can model the data $x_{1}, x_{2}, \ldots, x_{120}$ by using the model $x_{t}=$ $\left(-0.3807+0.6794 \varepsilon_{t}\right) x_{t-1}+\varepsilon_{t}$, where $\left\{\varepsilon_{t}\right\}$ is for i.i.d. random variables.

\section{Proofs of the main results}

In order to prove the main results, we need some auxiliary lemmas.

Lemma 5.1 Suppose that $\left(\mathrm{C}_{1}\right)-\left(\mathrm{C}_{3}\right)$ hold. Then, under $\mathrm{H}_{0}$, one-order generalized random coefficient autoregressive model (1.1) is $\beta$-mixing with geometric decaying order and $E Y_{t}^{3}<\infty$.

Proof The proof can be found in Carrasco and Chen [7].

Lemma 5.2 Suppose that $\left(\mathrm{C}_{1}\right)-\left(\mathrm{C}_{3}\right)$ hold. Then, under $\mathrm{H}_{0}$, for the one-order generalized random coefficient autoregressive model (1.1), there exists a positive constant $M$ such that, 
for any $0<d<3$,

$$
\limsup _{k \rightarrow \infty} \frac{\left|\sum_{t=1}^{k}\left(Y_{t}^{d}-E Y_{t}^{d}\right)\right|}{\sqrt{2 k \log \log k}} \leq M \quad \text { a.s. }
$$

Proof This lemma can be proved by Lemma 5.1 and Theorem 5 in Kuelbs and Philipp [23].

Lemma 5.3 Let $\left\{Y_{i}, i \geq 1\right\}$ be a stationary ergodic stochastic sequence with $E\left(Y_{i} \mid Y_{1}, Y_{2}\right.$, $\left.\ldots, Y_{i-1}\right)=0$ a.s. for all $i \geq 2$ and $E Y_{1}^{2}=1$. Then $\lim \sup _{n \rightarrow \infty} \sum_{i=1}^{n} \frac{Y_{i}}{(2 n \log \log n)^{\frac{1}{2}}}=1$ a.s.

Proof The proof can be found in Stout [24].

Proof of Lemma 2.3 Note that

$$
\left(\frac{1}{k} \sum_{t=1}^{k} N_{t} N_{t}^{\tau}\right)\left(\hat{\Upsilon}_{k}-\Upsilon\right)=\frac{1}{k} \sum_{t=1}^{k}\left(Y_{t}^{2}-N_{t}^{\tau} \Upsilon\right) N_{t}
$$

By the ergodic theorem, we have

$$
\frac{1}{k} \sum_{t=1}^{k} N_{t} N_{t}^{\tau} \stackrel{\text { a.s. }}{\rightarrow} E\left(N_{1} N_{1}^{\tau}\right)
$$

and

$$
\frac{1}{k} \sum_{t=1}^{k}\left(Y_{t}^{2}-N_{t}^{\tau} \Upsilon\right) N_{t} \stackrel{\text { a.s. }}{\rightarrow} 0
$$

These, together with (5.1), imply that $\hat{\Upsilon}_{k} \stackrel{\text { a.s. }}{\rightarrow} \Upsilon$, and thus the proof of Lemma 2.3 is complete.

Proof of Theorem 2.1 We apply Lemma 2.1 to prove Theorem 2.1.

Firstly we decompose $\hat{\phi}_{k}$ into the sum of martingale differences and a negligible term. Note that

$$
\begin{aligned}
\hat{\phi}_{k}= & \frac{\sum_{t=1}^{k} Y_{t} Y_{t-1}}{\sum_{t=1}^{k} Y_{t-1} Y_{t-1}}=\phi_{0}+\frac{\sum_{t=1}^{k} Y_{t} Y_{t-1}}{\sum_{t=1}^{k} Y_{t-1} Y_{t-1}}-\phi_{0}=\phi_{0}+\frac{\sum_{t=1}^{k}\left(Y_{t}-\phi_{0} Y_{t-1}\right) Y_{t-1}}{\sum_{t=1}^{k} Y_{t-1} Y_{t-1}} \\
= & \phi_{0}+\frac{\frac{1}{k} \sum_{t=1}^{k}\left(Y_{t}-\phi_{0} Y_{t-1}\right) Y_{t-1}}{E Y_{1}^{2}}+\frac{\frac{1}{k} \sum_{t=1}^{k}\left(Y_{t}-\phi_{0} Y_{t-1}\right) Y_{t-1}}{\frac{1}{k} \sum_{t=1}^{k} Y_{t-1} Y_{t-1}} \\
& -\frac{\frac{1}{k} \sum_{t=1}^{k}\left(Y_{t}-\phi_{0} Y_{t-1}\right) Y_{t-1}}{E Y_{1}^{2}} \\
= & \phi_{0}+\frac{\frac{1}{k} \sum_{t=1}^{n}\left(Y_{t}-\phi_{0} Y_{t-1}\right) Y_{t-1}}{E Y_{1}^{2}}-\left(\frac{1}{k} \sum_{t=1}^{k} Y_{t-1} Y_{t-1}\right)^{-1}\left(E Y_{1}^{2}\right)^{-1} \\
& \times\left(\frac{1}{k} \sum_{t=1}^{k} Y_{t-1} Y_{t-1}-E Y_{1}^{2}\right)\left(\frac{1}{k} \sum_{t=1}^{k}\left(Y_{t}-\phi_{0} Y_{t-1}\right) Y_{t-1}\right) .
\end{aligned}
$$


Next, we verify that they meet the conditions of Lemma 2.1.

Observe that $\left(E Y_{1}^{2}\right)^{-1}\left\{\left(Y_{t}-\phi_{0} Y_{t-1}\right) Y_{t-1}, t \geq 1\right\}$ is a sequence of mean zero stationary martingale difference. Thus, there exists a 1-dimensional standard Brownian motion $\left\{W_{1}(s), s \geq 0\right\}$ such that, for some $0<\lambda<\frac{1}{2}$,

$$
\sum_{t=1}^{k} E\left(Y_{1}^{2}\right)^{-1}\left(Y_{t}-\phi_{0} Y_{t-1}\right) Y_{t-1}-J^{-\frac{1}{2}} W_{1}(k)=O\left(k^{\lambda}\right) \quad \text { a.s. }
$$

In what follows, we prove that

$$
\sqrt{T} \sup _{k \geq T} \frac{\left(\frac{1}{k} \sum_{t=1}^{k} Y_{t-1} Y_{t-1}-E Y_{1}^{2}\right) \frac{1}{k} \sum_{t=1}^{k}\left(Y_{t}-\phi_{0} Y_{t-1}\right) Y_{t-1}}{\left(\frac{1}{k} \sum_{t=1}^{k} Y_{t-1} Y_{t-1}\right) E Y_{1}^{2}}=o_{p}(1) .
$$

By Lemma 4.3, we have

$$
\limsup _{k \rightarrow \infty} \frac{1}{(2 k \log \log k)^{\frac{1}{2}}} \sum_{i=1}^{k}\left(Y_{t}-\phi_{0} Y_{t-1}\right) Y_{t-1}=\sqrt{E\left(Y_{t}-\phi_{0} Y_{t-1}\right)^{2} Y_{t-1}^{2}} \quad \text { a.s. }
$$

From Lemma 4.2 we know that there exists a positive constant $M_{1}$ such that, for any $0<$ $d<3$,

$$
\limsup _{k \rightarrow \infty} \frac{\left|\sum_{t=1}^{k}\left(Y_{t}^{d}-E Y_{t}^{d}\right)\right|}{\sqrt{2 k \log \log k}} \leq M_{1} \quad \text { a.s. }
$$

from which, together with (5.6), we have

$$
\sqrt{k} \frac{\left(\frac{1}{k} \sum_{t=1}^{k} Y_{t-1} Y_{t-1}-E Y_{1}^{2}\right) \frac{1}{k} \sum_{t=1}^{k}\left(Y_{t}-\phi_{0} Y_{t-1}\right) Y_{t-1}}{\left(\frac{1}{k} \sum_{t=1}^{k} Y_{t-1} Y_{t-1}\right) E Y_{1}^{2}} \stackrel{\text { a.s. }}{\rightarrow} 0 \quad \text { as } k \rightarrow \infty
$$

Further, note that

$$
\begin{aligned}
\sqrt{T} \sup _{k \geq T} \frac{\left(\frac{1}{k} \sum_{t=1}^{k} Y_{t-1} Y_{t-1}-E Y_{1}^{2}\right) \frac{1}{k} \sum_{t=1}^{k}\left(Y_{t}-\phi_{0} Y_{t-1}\right) Y_{t-1}}{\left(\frac{1}{k} \sum_{t=1}^{k} Y_{t-1} Y_{t-1}\right) E Y_{1}^{2}} \\
\leq \sup _{k \geq T} \sqrt{k} \frac{\left(\frac{1}{k} \sum_{t=1}^{k} Y_{t-1} Y_{t-1}-E Y_{1}^{2}\right) \frac{1}{k} \sum_{t=1}^{k}\left(Y_{t}-\phi_{0} Y_{t-1}\right) Y_{t-1}}{\left(\frac{1}{k} \sum_{t=1}^{k} Y_{t-1} Y_{t-1}\right) E Y_{1}^{2}} .
\end{aligned}
$$

By (5.8), we prove (5.5). Thus, by Lemma 2.1, we prove Theorem 2.1 .

The authors declare that they have no competing interests.

\section{Authors' contributions}

All authors contributed equally to the writing of this paper. All authors read and approved the final manuscript.

\section{Author details}

${ }^{1}$ College of Mathematics, Jilin Normal University, Siping, 136000, P.R. China. ${ }^{2}$ College of Mathematics, Jilin University,

Changchun, 130012, P.R. China. ${ }^{3}$ Public Foreign Languages Department, Jilin Normal University, Siping, 136000, P.R. China. 


\section{Acknowledgements}

This work is supported by National Natural Science Foundation of China (Nos. 11271155, 11001105, 11071126, 10926156, 11071269), Specialized Research Fund for the Doctoral Program of Higher Education (Nos. 20110061110003,

20090061120037), Scientific Research Fund of Jilin University (Nos. 201100011, 200903278), the Science and Technology Development Program of Jilin Province (201201082), Jilin Province Social Science Fund (2012B115) and Jilin Province Natural Science Foundation (20101596, 20130101066JC).

Received: 9 March 2014 Accepted: 25 July 2014 Published: 21 Aug 2014

\section{References}

1. Hwang, SY, Basawa, IV: Parameter estimation for generalized random coefficient autoregressive processes. J. Stat. Plan. Inference 68, 323-327 (1998)

2. Tong, H: A note on a Markov bilinear stochastic process in discrete time. J. Time Ser. Anal. 2, 279-284 (1981)

3. Feigin, PD, Tweedie, RL: Random coefficient autoregressive processes: a Markov chain analysis of stationarity and finiteness of moments. J. Time Ser. Anal. 6, 1-14 (1985)

4. Hwang, SY, Basawa, IV: Asymptotic optimal inference for a class of nonlinear time series models. Stoch. Process. Appl. 46, 91-113 (1993)

5. Hwang, SY, Basawa, IV: The local asymptotic normality of a class of generalized random coefficient autoregressive processes. Stat. Probab. Lett. 34, 165-170 (1997)

6. Lee, S: Coefficient constancy test in a random coefficient autoregressive model. J. Stat. Plan. Inference 74, 93-101 (1998)

7. Carrasco, $M$, Chen, $X: \beta$-Mixing and moment properties of RCA models with application to $G A R C H(p, q)$. C. R. Acad. Sci., Sér. I Math. 331, 85-90 (2000)

8. Page, ES: Continuous inspection schemes. Biometrika 41, 100-114 (1954)

9. Page, ES: A test for a change in a parameter occurring at an unknown point. Biometrika 42, 523-527 (1955)

10. Page, ES: On problems in which a change in parameter occurs at an unknown point. Biometrika 44, 248-252 (1957)

11. Hinkley, DV: Inference about the change-point from cumulative sum tests. Biometrika 58, 509-523 (1971)

12. Brown, RL, Durbin, J, Evans, JM: Techniques for testing the constancy of regression relationships over time. J. R. Stat. Soc., Ser. B 37, 149-163 (1975)

13. Inclan, C, Tiao, GC: Use of cumulative sums of squares for retrospective detection of changes of variances. J. Am. Stat. Assoc. 89, 913-923 (1994)

14. Wichern, DW, Miller, RB, Hsu, DA: Changes of variance in first-order autoregressive time series models - with an application. J. R. Stat. Soc. Ser. C 25, 248-256 (1976)

15. Lee, $\mathrm{S}, \mathrm{Ha}, \mathrm{J}, \mathrm{Na}, \mathrm{O}, \mathrm{Na}$, S: The cusum test for parameter change in time series models. Scand. J. Stat. 30, 781-796 (2003)

16. Lee, S, Park, S: The monitoring test for the stability of regression models with nonstationary regressors. Econ. Lett. 105, 250-252 (2009)

17. Gombay, E, Serban, D: Monitoring parameter change in AR(p) time series models. J. Multivar. Anal. 100, 715-725 (2009)

18. Kang, J, Lee, S: Parameter change test for random coefficient integer-valued autoregressive processes with application to polio data analysis. J. Time Ser. Anal. 30, 239-258 (2009)

19. Na, O, Lee, J, Lee, S: Monitoring parameter change in time series models. Stat. Methods Appl. 20, 171-199 (2011)

20. Eberlein, E: On strong invariance principles under dependence assumptions. Ann. Probab. 14, 260-270 (1986)

21. Kuelbs, J, Philipp, W: Almost sure invariance principles for partial sums of mixing $B$-valued random variables. Ann. Probab. 8, 1003-1036 (1980)

22. Chu, CSJ, Stinchcombe, M, White, H: Monitoring structural change. Econometrica 64, 1045-1065 (1996)

23. Kuelbs, J, Philipp, W: Almost sure invariance principles for partial sums of mixing $B$-valued random variables. Ann. Probab. 8, 1003-1036 (1980)

24. Stout, WF: The Hartman-Wintner law of the iterated logarithm for martingales. Ann. Math. Stat. 41, 2158-2160 (1970)

10.1186/1029-242X-2014-309

Cite this article as: Zhao et al.: Test for parameter changes in generalized random coefficient autoregressive model. Journal of Inequalities and Applications 2014, 2014:309

\section{Submit your manuscript to a SpringerOpen ${ }^{\circ}$ journal and benefit from:}

- Convenient online submission

Rigorous peer review

- Immediate publication on acceptance

- Open access: articles freely available online

- High visibility within the field

- Retaining the copyright to your article 